\title{
Sistema Informatizado para Gerenciamento de Indicadores da Assistência de Enfermagem do Hospital São Paulo
}

\author{
COMPUTERIZED SYSTEM FOR MANAGING NURSING CARE INDICATORS AT HOSPITAL \\ SÃO PAULO
}

\section{SISTEMA INFORMATIZADO PARA GERENCIAMIENTO DE INDICADORES DE ATENCIÓN DE ENFERMERÍA DEL HOSPITAL SÃO PAULO}

\section{Lilian Lestingi Labbadia', Maria D'Innocenzo², Rosana Rodrigues Figueira Fogliano³, Gabriela Eneida Françolin Silva ${ }^{4}$, Rita Marina Ribeiro Melo de Queiroz ${ }^{5}$, Maria Isabel Sampaio Carmagnani ${ }^{6}$, Maria Elisabete Salvador ${ }^{7}$}

\section{RESUMO}

Os indicadores são instrumentos que possibilitam definir parâmetros que serão utilizados para realizar comparações e agregar juízo de valor frente ao encontrado e ao ideal estabelecido. Este estudo tem como objetivo descrever a experiência de um grupo de enfermeiras na criação do sistema informatizado desenvolvido no Hospital São Paulo para o gerenciamento de indicadores da assistência de enfermagem. A implantação deste sistema para o gerenciamento dos indicadores foi dividida em quatro etapas: desenvolvimento do manual de indicadores da assistência de enfermagem; realização de teste piloto registrado manualmente; desenvolvimento do sistema informatizado; implementação de estudo piloto do sistema informatizado em onze unidades do hospital.

\section{DESCRITORES}

Indicadores de Serviços

Indicadores de Qualidade em Assistência

à Saúde

Tecnologia da informação

Avaliação em enfermagem

\begin{abstract}
Indicators are tools that permit to define parameters that will be used to make comparisons between a result and its expected value, as well as to add a value of judgement in this regard. The purpose of this study is to describe the experience of a group of nurses in the development of a computerized system to manage nursing care indicators at Hospital São Paulo. Four stages were used to implement the indicator management system: developing a nursing care indicator handbook; performing a manually registered pilot test; developing the computerized system; and performing the pilot test of the computerized system in eleven units at the hospital.
\end{abstract}

\author{
DESCRIPTORS \\ Indicators of Health Services \\ Quality Indicators, Health Care \\ Information technology \\ Nursing assessment
}

\begin{abstract}
RESUMEN
Los indicadores son instrumentos que posibilitan definir parámetros que serán utilizados para realizar comparaciones y agregar juicio de valor frente a lo encontrado y al ideal establecido. Se objetivó describir la experiencia de un grupo de enfermeras en la implementación del sistema informatizado desarrollado en el Hospital San Pablo para el gerenciamiento de indicadores de atención de enfermería. Tal implantación para gerenciamiento de indicadores se dividió en cuatro etapas: desarrollo del manual de indicadores de atención de enfermería, realización de prueba piloto registrada manualmente, desarrollo de sistema informático e implementación de estudio piloto de sistema informático en once unidades del hospital.
\end{abstract}

\section{DESCRIPTORES}

Indicadores de Servicios

Indicadores de Calidad de la Atención de Salud

Tecnología de la información

Evaluación en enfermería

${ }^{1}$ Enfermeira. Mestre em Enfermagem pela Universidade Federal de São Paulo. Enfermeira do Departamento de Enfermagem da Universidade Federal de São Paulo. Membro do Grupo de Estudos e Pesquisa em Avaliação de Qualidade em Serviços de Saúde e Enfermagem da Universidade Federal de São Paulo. São Paulo, SP, Brasil. 1.labbadia@unifesp.br ${ }^{2}$ Enfermeira. Doutora em Enfermagem. Professora do Departamento de Enfermagem da Universidade Federal de São Paulo. Líder do Grupo de Estudos e Pesquisa em Avaliação de Qualidade em Serviços de Saúde e Enfermagem da Universidade Federal de São Paulo. São Paulo, SP, Brasil. mdinnocenzo@unifesp.br ${ }^{3}$ Enfermeira. Mestre em Enfermagem pela Universidade Federal de São Paulo. Gerente de Enfermagem das Clínicas Pediátricas do Hospital São Paulo. São Paulo, SP, Brasil. rosanafogliano@ajato.com.br ${ }^{4}$ Enfermeira. Especialista em Obstetrícia pela Universidade Federal de São Paulo e em Educação em Saúde pela Fundação Oswaldo Cruz. Responsável pelo Setor de Seleção e Treinamento de Pessoal pela Coordenadoria de Ensino e Pesquisa da Diretoria de Enfermagem do Hospital São Paulo. São Paulo, SP, Brasil. g.eneida@uol.com.br ${ }^{5}$ Enfermeira. Especialista em Epidemiologia Hospitalar pela Universidade de São Paulo. Gerente do Serviço de Enfermagem em Central de Desinfecção e Esterilização do Hospital São Paulo. São Paulo, SP, Brasil. rita.marina@unifesp.br ${ }^{6}$ Enfermeira. Doutora em Enfermagem pela Universidade Federal de São Paulo. Professora do Departamento de Enfermagem da Universidade Federal de São Paulo. Diretora de Enfermagem do Hospital São Paulo. São Paulo, SP, Brasil.carmagnani@unifesp.br ${ }^{7}$ Enfermeira. Doutora em Medicina pela Universidade Federal de São Paulo. Assessora da Diretoria de Enfermagem do Hospital São Paulo. São Paulo, SP, Brasil. betesalva@hotmail.com 


\section{INTRODUÇÃO}

Os enfermeiros, assim como os demais gestores que atuam na área da saúde, têm a responsabilidade de gerenciar seu serviço ou unidade, demonstrando os resultados obtidos por meio de indicadores ${ }^{(1)}$.

Vale ressaltar que os indicadores são instrumentos para definir parâmetros e realizar comparações, além de agregar juízo de valor frente ao encontrado e ao ideal estabelecido. Para tanto, é necessário que a fase de coleta e sistematização dos dados seja bem planeja$\mathrm{da}^{(2)}$. Ademais, informações convenientes e obtidas no momento certo podem beneficiar a administração dos serviços e favorecer a tomada de decisão com o menor grau de incerteza.

A melhoria da qualidade assistencial é considerada um processo amplo, dinâmico e exaustivo de identificação contínua dos fatores intervenientes do processo de trabalho da equipe de enfermagem. Requer do profissional enfermeiro a implantação de ações e a elaboração de instrumentos que possibilitem avaliar de maneira sistemática os níveis de qualidade dos cuidados prestados ${ }^{(3)}$.

No Brasil, os estudos têm contemplado a construção e validação dos indicadores de enfermagem, a exemplo do Núcleo de Apoio à Gestão Hospitalar subgrupo do Programa de Qualidade Hospitalar (NAGEH / CQH) ${ }^{(4-5)}$.

Mantido pela Associação Paulista de Medicina e Conselho Regional de Medicina do Estado de São Paulo, o NAGEH criou, no início dos anos 1990, o serviço de avaliação da qualidade da assistência prestada aos usuários de hospitais do Estado de São Paulo(6-7). Desde então, este Núcleo de pesquisa vem mobilizando os gestores de instituições hospitalares para a implantação e consolidação de medidas que promovam a qualidade dos serviços de saúde.

A utilização de novos métodos para prover a melhoria do serviço de enfermagem, somada ao considerável acervo de informações tanto assistencial quanto administrativo da enfermagem hospitalar, corroborou para a utilização da tecnologia da computação(4).

A informatização como forma de gerenciar, administrar, organizar, classificar, monitorar e obter informações relevantes, em tempo real, tornou o acesso aos indicadores de enfermagem mais dinâmico e produtivo( ${ }^{(8)}$.

A eficácia dos sistemas informatizados na área da saúde está amparada pela literatura e resultados práticos de instituições hospitalares que se tornaram referência mundial por utilizaram tais recursos ${ }^{(9)}$.

Neste cenário, autores de um estudo relataram sobre a importância de indicadores informatizados de enferma- gem considerando efetivas contribuições relacionadas à coleta, armazenamento e análise de dados, bem como o acesso ao sistema de forma intuitiva para os usuários finais. Este sistema apresenta-se em fase de implementação a fim de facilitar a mensuração sistemática e promover intervenções para melhoria da qualidade da assistência de enfermagem ${ }^{(10)}$.

A elaboração e aplicabilidade de indicadores de qualidade possuem estreita relação com a motivação e satisfação dos profissionais, repercutindo na assistência prestada aos usuários dos serviços de saúde ${ }^{(11)}$.

Nos processos avaliativos devem ser utilizadas informações válidas, pois somente com a aplicação de medidas validadas se evita correr o risco de distorcer o comportamento da equipe e, consequentemente, desmotivar os profissionais de enfermagem ${ }^{(12)}$.

Neste sentido, a Diretoria de Enfermagem do Hospital São Paulo (HSP) da Universidade Federal de São Paulo (UNIFESP) vem implantando novos métodos e moderna tecnologia para a criação e implantação de indicadores da assistência de enfermagem como forma de gerenciar o cuidado prestado nesta instituição.

Tendo por base quatro dos indicadores estabelecidos pelo NAGEH, foram desenvolvidos seis indicadores específicos e adaptados à realidade do Hospital São Paulo (HSP).

\section{OBJETIVO}

O presente trabalho objetiva descrever as etapas da construção do Sistema Informatizado de Indicadores da Assistência de Enfermagem do Hospital São Paulo.

\section{DESENVOLVIMENTO DO SISTEMA INFORMATIZADO}

A fim de coletar, gerenciar e avaliar dados, de forma rápida e eficaz, além de propor melhorias por meio de resultados, foi construído o Sistema Informatizado de Indicadores de Enfermagem do Hospital São Paulo (SIIE-HSP). Trata-se de um hospital universitário, com 742 leitos, sendo referência para alta complexidade e atendimento de 95\% para Sistema Único de Saúde.

O desenvolvimento do SIIE-HSP ocorreu no período de janeiro de 2005 à junho de 2008 e envolveu docentes do Departamento de Enfermagem da UNIFESP, profissionais do Departamento de Tecnologia da Informação da UNIFESP e enfermeiros da Diretoria de Enfermagem do HSP, após aprovação do Comitê de Ética e Pesquisa da instituição.

Dividido em quatro etapas, o desenvolvimento do SIIE-HSP ocorreu da seguinte forma:

Sistema Informatizado para Gerenciamento de Indicadores da Assistência de Enfermagem do Hospital São Paulo Labbadia LL, D'Innocenzo M, Fogliano RRF, Silva GEF, Queiroz RMRM, Carmagnani MIS, Salvador ME 
1. Criação e elaboração do Manual de Indicadores de Enfermagem: docentes do Departamento de Enfermagem da UNIFESP e enfermeiros do HSP desenvolveram o Manual de Indicadores de Enfermagem desta Instituição, baseado no Manual do NAGEH ${ }^{(7)}$ e nas experiências destes enfermeiros, utilizando técnica de grupo focal.

2. Realização de teste piloto: feito por meio de registros descritos em formulários elaborados pelo grupo de enfermeiros e testado em onze unidades do Hospital São Paulo, no período de março à abril de 2006.

3. Desenvolvimento do SIIE-HSP: Docentes e Enfermeiros do Grupo de Melhoria de Processos da Assistência de Enfermagem - Qualidade da Assistência de Enfermagem do Hospital São Paulo - e analistas de sistemas do Departamento de Tecnologia da Informação da UNIFESP desenvolveram e testaram o SIIE-HSP no período de julho de 2007 à maio de 2008.

4. Implementação de estudo piloto do SIIE-HSP: a capacitação para a utilização do SIIE-HSP foi realizada no Laboratório de Informática da instituição, com carga horária de 4h/enfermeira, em junho de 2008, para 60 enfermeiras de todos os turnos de dez unidades de internação do HSP.

Desenvolvido com a linguagem de programação para bancos de dados conhecida como PHP - Hypertext Preprocessor e sob a plataforma de sistema para gerenciar banco de dados Oracle Corporation 10g, o SIIE-HSP foi integrado ao Sistema de Internação de Pacientes desta Instituição com acesso pelos computadores das unidades do HSP.

O SIIE-HSP contém os indicadores que envolvem diretamente os eventos adversos que podem ocorrer durante os procedimentos de assistência prestada ao paciente pela equipe de enfermagem: Extubação não Planejada,
Hipotermia, Lesões de Pele, Perda de Cateter Urinário, Perda de Cateter Vascular Central, Perda de Cateter Vascular Periférico, Perda de Dreno, Perda de Sonda do Trato Gastro Intestinal, Queda de Paciente e Úlcera por Pressão.

O SIIE-HSP emite também relatórios que possibilita a identificação dos eventos adversos por paciente, série histórica por unidade e incidência de queda e úlcera por pressão com identificação e classificação do risco (Figuras 1, 2, 3 e 4).

\begin{tabular}{|c|c|c|}
\hline \multicolumn{3}{|c|}{$\begin{array}{l}\text { Unidade de Internaçios Ur coNvêNio } 9 \text { ANDAR } \\
\text { Datas 04/06/2000 } \\
\text { Total de pacientes internadosa } 6\end{array}$} \\
\hline \multicolumn{2}{|l|}{ Ptens } & Qtede \\
\hline \multicolumn{2}{|l|}{ Eteter vascular perifterico } & 4 \\
\hline \multicolumn{2}{|l|}{ Coteter vascular central } & 0 \\
\hline \multicolumn{2}{|l|}{ Lesto de pele (caso novo) } & 0 \\
\hline \multicolumn{2}{|c|}{ Paciente com risco para UP (escala de Braden < 10) } & 0 \\
\hline \multicolumn{2}{|l|}{ alliasinos riaco } & 0 \\
\hline \multicolumn{2}{|l|}{ atho risco } & 0 \\
\hline \multicolumn{2}{|l|}{ risco moderado } & b \\
\hline \multicolumn{2}{|l|}{ baixo risce } & 0 \\
\hline \multicolumn{2}{|l|}{ sem risco } & 6 \\
\hline \multicolumn{2}{|l|}{ Paciente com risco para queda } & 4 \\
\hline \multicolumn{2}{|l|}{ allo risco } & 2 \\
\hline \multicolumn{2}{|l|}{ risco } & 2 \\
\hline \multicolumn{2}{|l|}{ sem risco } & 2 \\
\hline \multicolumn{2}{|l|}{ Coteter urinirio } & 0 \\
\hline \multicolumn{2}{|l|}{ Sonda do TGI } & 0 \\
\hline \multicolumn{2}{|l|}{ Dreno } & 0 \\
\hline \multicolumn{2}{|l|}{ Paciente com hipotermis } & 0 \\
\hline \multicolumn{2}{|l|}{ Paciente intubudo/fraqueotlomizudo } & 1 \\
\hline \multicolumn{3}{|c|}{ Cadastro: 04/06/2008 08129 TREINA } \\
\hline
\end{tabular}

Figura 1 - Tela do SIIE-HSP: Dados relacionados aos pacientes por unidade de internação

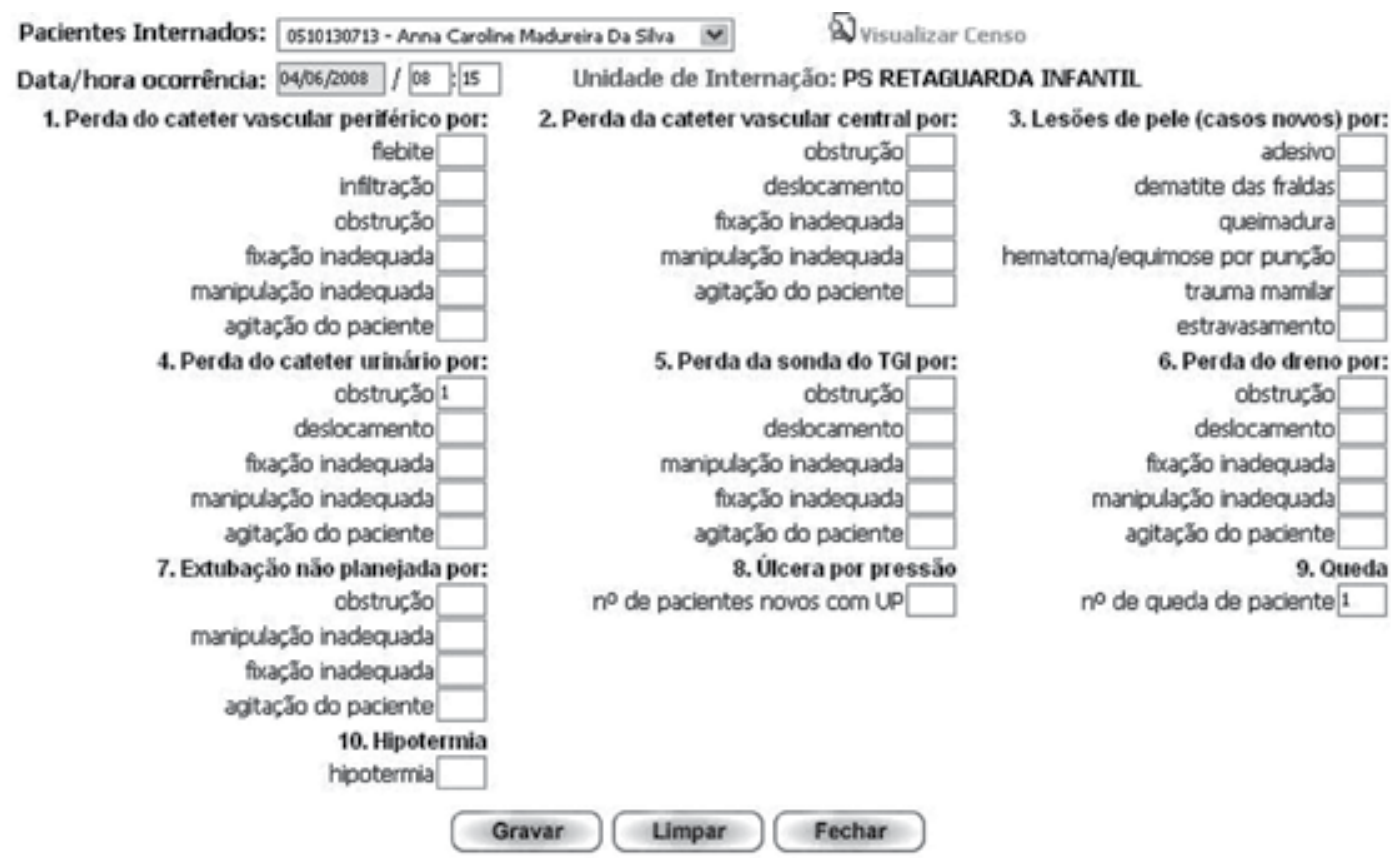

Figura 2 - Tela do SIIE-HSP: Relato de ocorrências por paciente 
Hospital São Paulo - Diretoria de Enfermagem

Sistema de Indicadores da Assistência de Enfermagem

Relato de Ocorrência de Queda

Fatores de risco para queda identificados durante a internação?

- Idade $>=75$ anos

- Uso de sedativo ou pós - sedativos

\section{- Dificuldade de march.}

Sim NẼo Quais?

- Moblidade fisica prejudicada

\section{Descrição do evento:}

tentou sair da cama por babixo da grade e calu.

\section{Ação Corretiva:}

Avaliaçẫo de enfermagem:

nö̉ apresenta lesöes aparentes, solicilada avaliaçäo médica.

Exames / procedimentos realizados:

RX sem sinal de fratura. Tomo sem sinal de lesöes.

Figura 3 - Tela do SIIE-HSP: Relato de ocorrência de queda

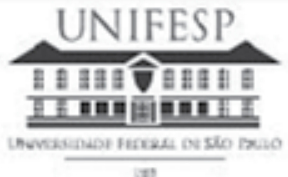

Hospital Săo Paulo - Diretoria de Enfermagem

Relatório dos Indicadores da assistência de enfermagem

Unidade de Internação: U Observação Ps Adulto

Período de coleta: 01/01/2008 a 30/05/2008

Perda do cateter vascular periférico por:
1) flebite
2) infiltração
3) obstruçã̃o
4) fixação inadequada
5) manipulação inadequada
6) agitação do paciente

\section{Queda}

1) no de queda de paciente

$$
50 \% \quad \frac{\text { no de perdas de cateteres vasculares periféricos }}{n^{0} \text { de pacientes com cateteres vasculares perifféricos-dia }} \times 100
$$

$13.64 \%$

no de quedas

no de pacientes-dis

Figura 4 - Tela do SIIE-HSP: Relatório dos Indicadores da Assistência de Enfermagem 


\section{CONCLUSÃO}

Reunindo muitos benefícios, o Sistema Informatizado de Indicadores de Enfermagem do Hospital São Paulo destaca-se por armazenar dados pertinentes aos processos assistenciais de enfermagem e disponibilizá-los para a avaliação dos resultados da assistência de enfermagem. Estes poderão ser consultados em tempo real e impressos no momento necessário.

Tais recursos poderão ser utilizados, em virtude do desenvolvimento informatizado, por meio de bancos de dados interativo, interface intuitiva, de fácil manuseio e atrativa do ponto de vista pedagógico, tendo o enfermeiro uma moderna ferramenta de trabalho, capaz de mensurar e contribuir para a qualidade de seu serviço.

Contudo, a programação do SIIE-HSP representou um desafio em termos de desenvolvimento, em virtude da falta de modelos informatizados que proporcionasse um banco de dados livre de erros de operacionalidade e que refletisse a realidade da instituição.

No entanto, serão necessários mais estudos a fim de avaliar e comprovar a eficácia deste Sistema, analisar a sua utilização por parte da equipe de enfermagem e sensibilizá-la quanto à importância da utilização dos indicadores no processo de trabalho, contribuindo para a melhoria contínua do cuidado ao paciente.

\section{REFERÊNCIAS}

1. Bohomol E. Padrões para avaliação da qualidade da assistência de enfermagem. In: Innocenzo M, organizadora. Indicadores, auditorias e certificações: ferramentas de qualidade para gestão em saúde. São Paulo: Martinari; 2006. p. 73-83.

2. Carvalho G, Rosemburg CP, Buralli KO. Avaliação de ações e serviços de saúde. Mundo Saúde. 2000;24(1):72-88.

3. Fonseca AS, Yamanaka NMA, Barison T, Luz SF. Auditoria e o uso de indicadores assistenciais: uma relação mais que necessária para a gestão assistencial na atividade hospitalar. Mundo Saúde. 2005;29(2):161-8.

4. Reis EAA, Denser CPAC, Minatel VF, Bork AMT. Definição de Indicadores de Assistência de Enfermagem a partir dos dados mínimos [Internet]. [citado 2008 jun. 21]. Disponível em: http://www.sbis.org.br/cbis9/arquivos/730.doc

5. Tronchin DMR, Melleiro MM, Mota NVYP. Indicadores de qualidade de enfermagem: uma experiência compartilhada entre instituições integrantes do Programa de Qualidade Hospitalar. Mundo Saúde. 2006;30(2):305.

6. Mota NVP, Melleiro MM, Tronchin DMR. A construção de indicadores de qualidade de enfermagem: relato da experiência do Programa de Qualidade Hospitalar. Rev Adm Saúde. 2007;9(34):9-15.
7. Programa de Qualidade Hospitalar (CQH). Manual de Indicadores de Enfermagem NAGEH [Internet]. São Paulo: APM/ CREMESP; 2006 [citado 2009 jul. 12]. Disponível em: http:// www.cqh.org.br/files/Manual\%20de\%20Indicadores\%20NAGEH\%20-\%20V.FINAL.pdf

8. Schout D, Novaes HMD. Do registro ao indicador: gestão da produção da informação assistencial nos hospitais. Ciênc Saúde Coletiva. 2007;12(4):935-44.

9. Caliri MHL. Usando os recursos da internet na enfermagem. Rev Latino Am Enferm. 1997;5(1):98-100.

10. Cohen R, Fish M, Peri H, Niv G, Kedem K, Nir E, et al. "When quality and computers meet": construction of a computerized nursing quality indicators' scale for departmental self-monitoring and improvement of quality of care. Stud Health Technol Inform. 2009; 146:414-5.

11. Kurcgant $P$, Tronchin DMR, Melleiro MM. A construção de indicadores de qualidade para a avaliação de recursos humanos nos serviços de enfermagem: pressupostos teóricos. Acta Paul Enferm. 2006;19(1):88-91.

12. Vituri DW, Matsuda LM. Content validation of quality indicators for nursing care evaluation. Rev Esc Enferm USP [Internet]. 2009 [cited 2009 July 12];43(2):429-37. Available from: http://www.scielo.br/pdf/reeusp/v43n2/en_a24v43n2.pdf 